\title{
A MACHINE-LEARNING MODEL FOR THE DETERMINATION OF MACRO-SCALE MASONRY PROPERTIES BASED ON A VIRTUAL LABORATORY AT MICRO-SCALE
}

\author{
PHILIP KALKBRENNER ${ }^{1}$, LUCA PELÀ ${ }^{1}$ AND RICCARDO ROSSI ${ }^{1}$ \\ ${ }^{1}$ Department of Civil and Environmental Engineering \\ Universitat Politécnica de Catalunya (UPC-BarcelonaTech) \\ 08034 Barcelona, Spain \\ e-mail: philip.kalkbrenner@upc.edu, luca.pela@upc.edu, rrossi@cimne.upc.edu
}

Keywords: Machine-learning, Historical Structure, Masonry, Virtual Laboratory, Representative Volume Element, Micro-scale, Macro-scale, Homogenization Technique

\begin{abstract}
Cutting-edge methods in the computational analysis of structures have been developed over the last decades. Such modern tools are helpful to assess the safety of existing buildings. Multi-scale techniques have been proposed to combine the accuracy of micromodelling and the computational efficiency of macro-modelling. Machine-learning tools have been utilized successfully to train specific models by feeding big source data from different fields, e.g. autonomous driving, face recognition, etc. This research proposes a continuous nonlinear material law that can reproduce data from micro-scale analysis. The proposed method is based on a machine-learning tool that links the two scales of the analysis by training a macro-model smeared damage constitutive law through benchmark data from numerical tests derived from micro-models.
\end{abstract}

\section{INTRODUCTION}

Building with masonry is one of the oldest construction techniques and a majority of the building stock worldwide is constructed using this component material. Due to its social and cultural value the construction type and assessment tools for masonry must be preserved.

Nowadays numerical methods are considered as a commodity in structural engineering. Computer analysis techniques have been proved successful in assessing the complex behaviour of masonry structures. Two main lines of research can be identified, each with benefits and drawbacks: macro-modelling and micro-modelling.

Masonry is a heterogeneous material composed of brick units and mortar joints. Each material has different properties. Micro-scale modelling approaches, which are based on the idea of distinguishing between the masonry components, have been proved to be very accurate [1 - 3]. The associated modelling and computation cost is however too high in application to large structures. Macro-modelling [4 - 7] represents the masonry material as a homogenized continuum and thus offers wide applicability to real scale structures. The weakness of macro-modelling approaches is intrinsic to the idea of smearing the behaviours of the masonry components into a single continuum. The advantage is of course that the small 
computational cost allows the modelling of large scale structures.

Given both the advantages and disadvantages of the modelling techniques, multi-scale modelling approach has the aim to provide a direct relationship between both scales. This approach requires a homogenization technique to scale strain and stress states from the micro to the macro level. Petracca et al. [8] have analysed the classical first order computational homogenization as an advanced technique applied to the analysis of masonry structures.

This approach consists of three steps, as defined in [8]. Down-scaling transfers the strains from the macro scale and applies them as boundary conditions to a representative volume element (RVE) at the micro-scale. Solution of the boundary value problem consists in solving the RVE analysis at micro-scale. Up-scaling transfers the stresses from micro-scale to macroscale by computing a volume average of the micro-scale stresses. The described procedure has been carried out successfully and gives accurate results when compared with experimental tests on masonry structures $[8,9]$.

However, performing the homogenization at each gauss point of the macro scale model brings along a huge computational effort. Hence, an appropriate alternative to the classical computational homogenization that disconnects the above described steps must be found. A recent investigation presents the concept of smart constitutive laws trained by machinelearning tools [10]. The outcome of such models is a constitutive model that can be applied for numerical analysis of heterogeneous materials in terms of macro scale analysis.

The motivation of the present investigation is to train a machine-learning model that transfers the complex micro-structural behaviour of masonry to a macro model approach. Benchmark data for the nonlinear tests on maso

The paper is organ procedure. Section 3 su optimization procedure and application to the present constitutive law. Section 5 explains the he madhinc-learning trai
nized as follows. Sedticro-models in a
ummarizes the constitutive
ing model. Section 4
and application to the

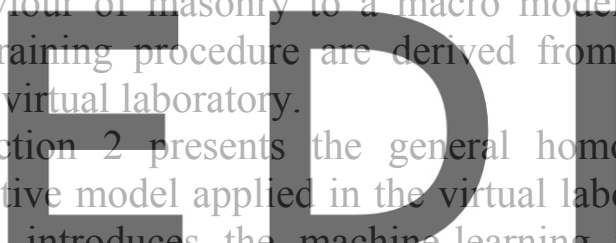
mogenization

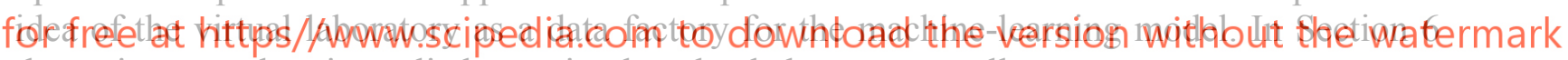
the entire procedure is applied to an in plane loaded masonry wall.

\section{THE MACHINE-LEARNING COMPUTATIONAL HOMOGENIZATION TECHNIQUE}

"Classical" computational homogenization techniques like first order computational homogenization allow representing the microscopic behaviour of the heterogeneous masonry material very accurately. Such techniques are based on utilizing representative volume elements (RVEs) in order to analyse the material's micro mechanical behaviour. However, they still do not disconnect the micro and the macro-scale at the solving stage. When used together with the Finite Element Method, the procedure transforms strains at the gauss point of the macro-scale into a boundary value problem of the RVE at the micro scale. Thus, a boundary value problem of an entire RVE is solved in order to obtain stress results for only one gauss point at the macro-scale. The computational cost becomes tremendous, the larger the macro-scale models are.

Researches made in [11] introduce an off-line technique to avoid solving the RVE at each analysis step. The strains at the macro scales are not sent to a micro model RVE, but to a large 
database. The creation of this database includes the strain and stress results of previously performed analyses on a RVE. Thus, the strains coming from the macro-scale can "choose" their corresponding stress state from the database by comparing the incoming strains with the ones of the database. The works made in [11] contribute substantially to the approach of the present investigations, since a virtual laboratory is used to construct the database. However, the method still implies jumping away from the macro-scale at the solving stage.

The idea of the present research is to avoid hopping around at multiple scales. The key issue is then finding a single homogeneous continuum damage model for the macro scale analysis of masonry that takes into account the masonry's heterogeneity. This research focuses on utilizing a machine-learning model to train the parameters of such a homogeneous continuum damage model for masonry structures. The following sections give an overview of the novel homogenization technique that consists of the following main steps. First, the derivation of a machine-learning model including a homogeneous constitutive law. Second, the construction of a virtual laboratory as a training data factory by running multiple tests on a representative volume element. Third, training and evaluation of the machine-learning model parameters. The fundamental ideas behind and the proper application of each step is presented in the following three sections.

\section{CONSTITUTIVE MODEL}

\subsection{Constitutive Law}

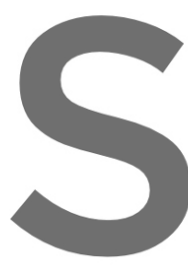
According with the theory is adopted to distingui based on the principle and undamaged conf guration behaviour [12].
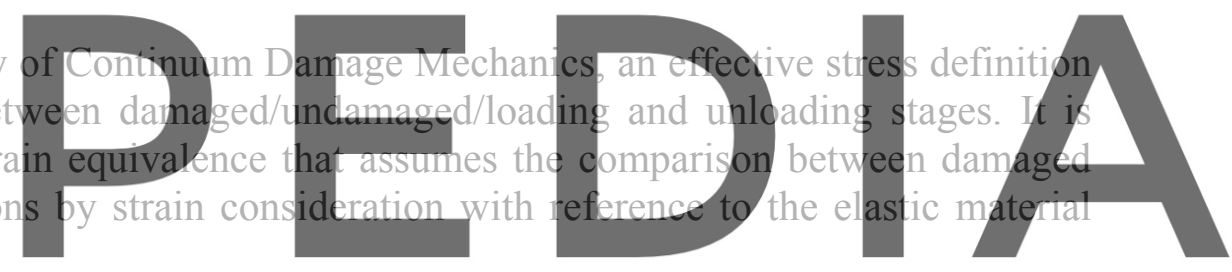

Register for free at https//www.scipedia.com to $\overline{\bar{\sigma}} C_{\text {dów }}{ }^{\epsilon}$ nload the version without the watermark

Where $\bar{\sigma}$ is the effective stress tensor, $\epsilon$ the strain tensor and $C$ is the fourth order elasticity tensor. The works of [13 - 15] introduce separated internal damage variables to use damage scalar models for tensile and compressive stress contributions and define the stress tensor $\boldsymbol{\sigma}$ as follows

$$
\boldsymbol{\sigma}=\left(1-d^{+}\right) \overline{\boldsymbol{\sigma}}^{+}+\left(1-d^{-}\right) \overline{\boldsymbol{\sigma}}^{-}
$$

The internal damage variables $d^{+}$and $d^{-}$indicate the grade of damage in tension and compression, respectively $\left(d^{ \pm} \in[0,1]\right)$. The tensors $\overline{\boldsymbol{\sigma}}^{+}$and $\overline{\boldsymbol{\sigma}}^{-}$are the positive and negative parts of the effective stress tensor and account for different nonlinear behaviours in tension and compression. The decomposition of the effective stress tensor $\overline{\boldsymbol{\sigma}}$ is performed according to $[15,16]$.

\subsection{Yield Criteria}

Lubliner et al. [17] proposed a proper yield criterion that considers two scalar values that reflect different material behaviours in tension and compression. The values $\tau^{+}$(tension) and $\tau^{-}$(compression) indicate the equivalent uniaxial stress. This research includes a modified 
Lubliner yield criterion [8] in compression and a Rankine yield criterion in tension. The scalars are then defined as follows

$$
\begin{gathered}
\tau^{+}=\left\langle\bar{\sigma}_{\max }\right\rangle \text { (Rankine) } \\
\tau^{-}=H\left(-\bar{\sigma}_{\text {min }}\right)\left[\frac{1}{1-\alpha}\left(\alpha \bar{I}_{1}+\sqrt{3 \bar{J}_{2}}+\kappa \beta\left\langle\bar{\sigma}_{\text {max }}\right\rangle\right)\right]
\end{gathered}
$$

The constant $\kappa$ aims to better control the effect of the compression surface on the shear strength of the model. It leads from 0 ( $\rightarrow$ Drucker-Prager surface) to 1 ( $\rightarrow$ Lubliner surface). To better understand the meaning of each entity in the equations, the authors advise to read carefully the explanations made in [3].

\subsection{Damage Evolution}

A threshold value must be introduced to distinguish between an undamaged and a damaged state of the material. At the same time, the threshold must indicate if the model undergoes loading/unloading or reloading, since the damage is an irreversible process. Thus, two additional scalar values are introduced. The actual threshold $r_{n}^{ \pm}$is defined as follows

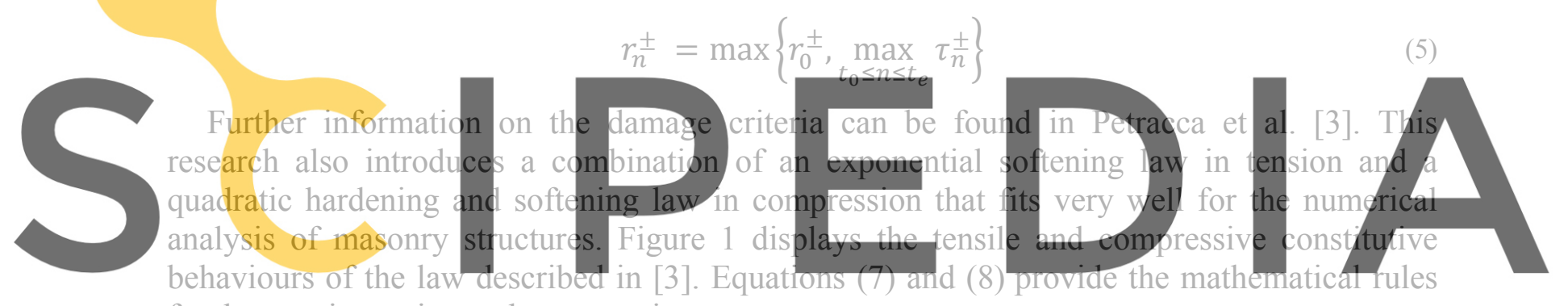

for damage in tension and compression.

Register for free at https//www.scipedia.com to download the version without the watermark

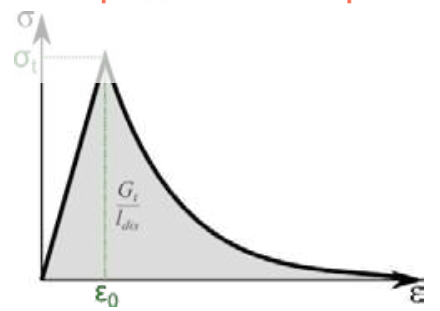

(a)

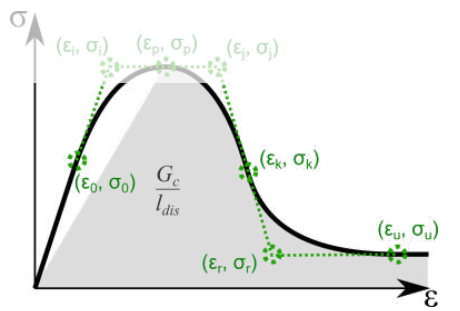

(b)

Figure 1: Uniaxial damage laws for (a) exponential softening and (b) quadratic hardening and softening

$$
\begin{gathered}
d^{-}\left(r^{-}\right)=1-\frac{\Psi\left(\xi^{-}\right)}{r^{-}} \\
d^{+}\left(r^{+}\right)=1-\frac{r_{0}^{+}}{r^{+}} \exp \left\{2 H_{\text {dis }}\left(\frac{r_{0}^{+}-r^{+}}{r_{0}^{+}}\right)\right\}
\end{gathered}
$$

Where $\Psi\left(\xi^{-}\right)$depends on the damaged state (hardening or softening) and on a strain-like counterpart $\xi^{-}$. A detailed description of $\Psi\left(\xi^{-}\right)$, and the energy regularization in tension and 
compression can be found in reference [3].

\section{THE SUPERVISED MACHINE-LEARNING MODEL}

\subsection{Fundamentals}

Machine-learning tools are very useful to support computations when a certain task is extremely difficult to program. The general idea is to collect data and utilize them to train a model to solve a task. This way of learning connects a set of inputs $I_{\text {true }}$ and a set of outputs $O_{\text {true }}$ by searching a function $F$ so that $F\left(I_{\text {true }}\right)=O_{\text {true }}$. The function aims to fit the inputs to the outputs.

The goal of such a fitting is to adjust the machine-learning model parameters $\boldsymbol{\Theta}$ so that the given inputs $I_{\text {true }}$ fit to the given outputs $O_{\text {true }}$ as accurately as possible. The given outputs $O_{\text {true }}$ are compared with predicted outputs $O_{\text {pred }}$ in a loss function $\mathcal{L}(\boldsymbol{\Theta})$. If the loss function does not fulfill a predefined loss minimum, an adjustment of the models parameters $\Theta$ is carried out to approximate the outputs $O_{\text {pred }}$ of the model to the given outputs $O_{\text {true }}$.

The most popular algorithms to perform such optimizations are gradient descent operators. A detailed overview is given in [18]. The present research applies the Adam optimizer [19].

The loss function counts the machine-learning prediction error. A classical computation of the loss is the $\mathcal{L}_{2}$ loss function that computes a mean square error. The entire loss function applied to the gradient descent optimization for a batch of inputs and outputs of size $m$ can be
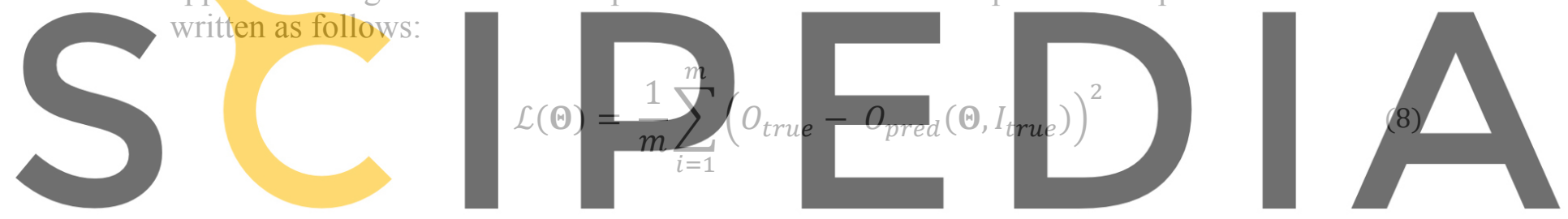

4.2 Present Application Procedure

Register for free atthttps//www scipedia, com to download the version without the watermark

this research. In order to apply the machine-learning approach its procedure must be mapped to the present scientific issue. Thus the fundamental questions are what are the coupled input and output items, and which mathematical formulation connects them.

- The function $F\left(I_{\text {true }}\right)$ : This research aims to find a correlation between strains and stresses for a homogenized material. For any material, constitutive laws can define such a correlation. Thus the here considered mathematical formulation for the machine-learning model is derived from a constitutive law and is defined as function $\boldsymbol{\Gamma}\left(I_{\text {true }}\right)$.

- The input $I_{\text {true }}$ and output $O_{\text {true }}$ : The constitutive law presented in Section 3 is based on strain equivalence. Then the input $I_{\text {true }}$ of the function is a set of strain states $\boldsymbol{\varepsilon}_{\text {true }}$. In order to perform the supervised learning, the model requires the reference true output stresses $\sigma_{\text {true }}$.

- The parameters $\boldsymbol{\Theta}$ : The strains $\boldsymbol{\varepsilon}_{\text {true }}$ run through the model and are adjusted by the constitutive law material parameters which act as the trainable variables of the machinelearning model.

- The predicted output $O_{\text {pred }}$ : The predicted output $O_{\text {pred }}$ of the machine-learning model are the stresses $\boldsymbol{\sigma}_{\text {pred }}$ computed by the constitutive law. 
After having introduced the machine-learning model's participating entities, the general mathematical formulation of the optimization process can be stated as follows:

$$
\boldsymbol{\Gamma}\left(\boldsymbol{\varepsilon}_{\text {true }}, \boldsymbol{\Theta}\right) \approx \boldsymbol{\sigma}_{\text {true }}
$$

The model searches a modification of the parameters $\boldsymbol{\Theta}$ of the constitutive model $\boldsymbol{\Gamma}$, so that by inputting strain states $\boldsymbol{\varepsilon}_{\text {true }}$ an accurate approximation to the reference stresses $\boldsymbol{\sigma}_{\text {true }}$ can be achieved. A predefined learning criterion checks the error value of the actual optimization state. If the error value is less or equal to the learning criterion, the optimization finishes and stores the actual modification of the parameters $\boldsymbol{\Theta}$ as the optimized model parameters $\boldsymbol{\Theta}^{*}$. Then the model can be utilized to predict stresses $\boldsymbol{\sigma}_{\text {pred }}$ by entering any strain state $\tilde{\boldsymbol{\varepsilon}}$. It follows:

$$
\boldsymbol{\Gamma}\left(\tilde{\boldsymbol{\varepsilon}}, \boldsymbol{\Theta}^{*}\right)=\boldsymbol{\sigma}_{\text {pred }}
$$

The implementation of the machine-learning model takes place in the python-based open source framework TensorFlow [20]. It offers a variety of already implemented optimizers including the above mentioned. The machine-learning model flow inside TensorFlow is based on a computation graph consisting of nodes where each node performs a mathematical operation.

VIRTUAL LABORATORY

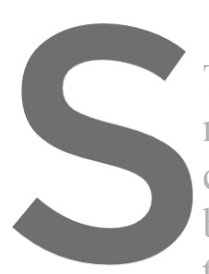

The here presented Thus, the material's heterogeneity must be repres into account th
model. Such a model takes in
components. It is called a representative volun
boundary conditions, in order to analyse the $\mathrm{h}$ this behaviour is essential for any homogenization technique.
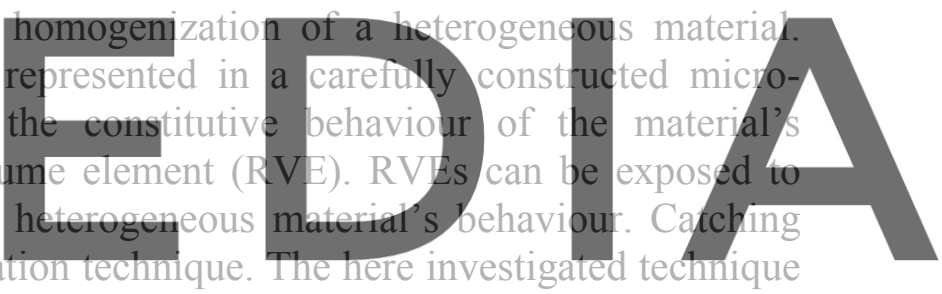
necessitates a space where boundary conditions can be applied to a properly micro-modelled

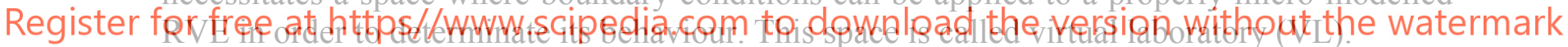

The accuracy of the machine-learning homogenization technique is based on two operational needs: a) a large amount of data and b) a broad data representation. Need a) is crucial for any machine-learning model: the more input data in learning, the more accurate its prediction. Need b) is crucial for the homogenization technique. The data should represent the nonlinear and heterogeneous behaviour of the RVE in order to be able to consider it in the homogenization technique. This can be achieved by performing the virtual experiment up to failure and by carrying out multiple virtual experiments on the same RVE, each taking into account a different boundary condition. This leads to a variety of deformations of the RVE.

\subsection{The Boundary Value Problem of the Representative Volume Element}

This Section explains the fundamental part of the VL: the representative volume element (RVE) and the solution of its boundary value problem. It is illustrated for a two dimensional RVE but can analogously be applied to a three dimensional one. This work applies the Finite Element (FE) method as numerical tool. Figure 2 shows the schematic view of a micro model RVE of a masonry wall. 


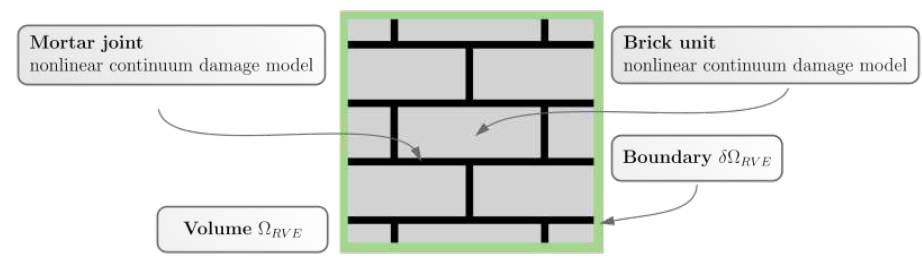

Figure 2: Example of a micro-scale modelled representative volume element $\boldsymbol{\Omega}_{\boldsymbol{R} V \boldsymbol{E}}$ for the virtual laboratory with boundary $\partial \boldsymbol{\Omega}_{R V E}$

In order to solve the boundary value problem of the RVE, boundary conditions must be defined. This can be done by applying a displacement fluctuation field to the RVE. The research done in [8] summarizes several fluctuation fields that can be implemented to RVE analyses. In the present monotonically increasing displacements are applied to the RVE's boundary $\partial \Omega_{R V E}$ as follows:

$$
d_{x}=\left(\varepsilon_{x x} \cdot x+\varepsilon_{x y} \cdot y\right) \cdot t, \quad \quad d_{y}=\left(\varepsilon_{y y} \cdot y+\varepsilon_{x y} \cdot x\right) \cdot t
$$

Where $d_{x}$ and $d_{y}$ are the displacements applied to the FE nodes of the RVE's boundary in $\mathrm{x}$ - and $\mathrm{y}$-direction, respectively. The factor $t \in\left[t_{0}, t_{e}\right]$ describes the time instance of the actual analysis step and increases monotonically. The constants $\varepsilon_{x x}, \varepsilon_{y y}$ and $\varepsilon_{x y}$ are the components of a previously defined strain state $\varepsilon$ in Voigt's notation. Modifying them allows performing a variety of virtual experiments. Investigations made by Zaghi et al. [11] show that all possible strain states can a Cartesian coordinate system
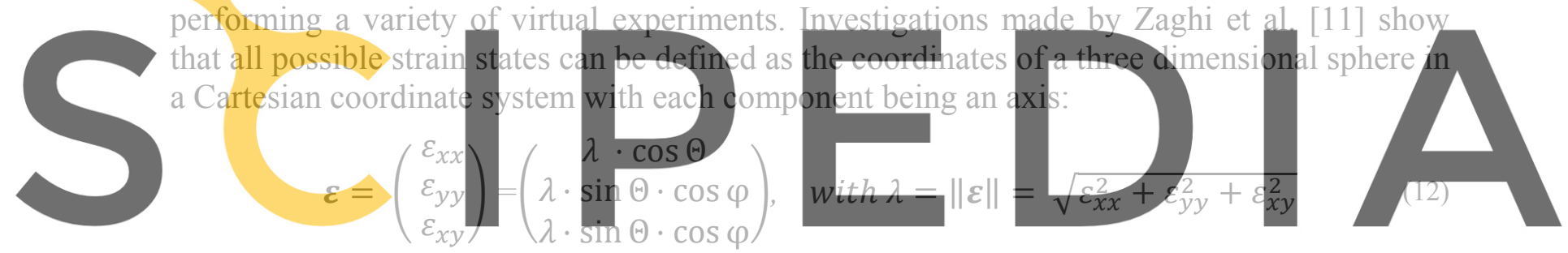

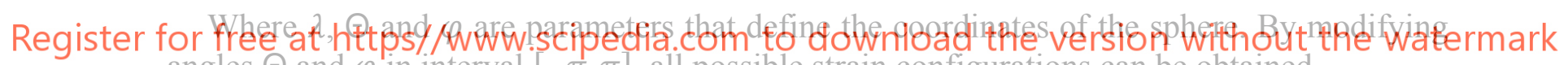
angles $\Theta$ and $\varphi$ in interval $[-\pi, \pi]$, all possible strain configurations can be obtained.

A numerical non-linear finite element analysis solves the boundary value problem for each virtual experiment. The open source framework KRATOS Multiphysics [21] is utilized. It enables to program a loop over all the considered cases in the virtual laboratory.

While solving the boundary value problem, a procedure ensures to store the stresses at each gauss point of the analysis. The transition from micro to macro-scale takes place by computing an average of the saved RVE stresses at each step. The applied general computation of the up-scaled stress $\widehat{\boldsymbol{\sigma}}$ for two-dimensional elements is as follows:

$$
\widehat{\boldsymbol{\sigma}}=\frac{1}{A_{R V E}} \sum_{i=1}^{n}\left[\frac{A_{i}}{k_{i}} \sum_{j=1}^{k_{i}} \boldsymbol{\sigma}_{i, j}\right]
$$

Where $n$ is the total number of elements of the RVE, $A_{R V E}$ is the area of the total RVE, $A_{i}$ is the area of the $i$-th element, $k_{i}$ is the number of gauss points of the $i$-th element, $\sigma_{i, j}$ is the stress vector of the $i$-th element at its $j$-th gauss point. The corresponding up-scaled strain $\hat{\boldsymbol{\varepsilon}}$ can be derived by transforming the applied boundary strain $\varepsilon$ to the engineering notation. 


\subsection{Isotropic Mapping of Up-scaled States}

The introduced homogenization technique includes the training of an isotropic constitutive law. Thus an isotropic relation between the up-scaled strain $\hat{\boldsymbol{\varepsilon}}$ and the up-scaled stress $\widehat{\boldsymbol{\sigma}}$ must be ensured. This is not the case if stresses of a non-isotropic RVE are averaged. In this research the assumption holds, that the elasticity between both the up-scaled entities from the RVE follows an orthotropic relation.

Pelà et al. [6] apply a concept of mapped stress tensor to the nonlinear damage analysis of masonry structures. The idea is that a linear mapping can be found which transforms an initially anisotropic behaviour into an isotropic one. Provided such mapping is known, one can map the input strain onto the isotropic space, operate onto it to obtain an isotropic stress and then inverse map the obtained stress to obtain an equivalent anisotropic behaviour.

This is best explained in symbols, by providing a technique for the construction of the mapping operator. Let $\boldsymbol{C}$ be a generic elasticity tensor and $\boldsymbol{C}^{*}$ the closest isotropic tensor (see e.g. [22] for a discussion on the determination of $\boldsymbol{C}^{*}$ ). Provided that $\boldsymbol{C}$ is Symmetric Positive Definite (SPD), something that should be guaranteed for any elasticity tensor. A linear operator $\mathbf{T}$ can be found such that $\mathbf{C}=\boldsymbol{T}^{t} \boldsymbol{C}^{*} \boldsymbol{T}$. A simple constructive proof of this can be found in [23]. Since $\sigma=C: \epsilon$ it follows that $\sigma=T^{t} C^{*} T \epsilon$. Premultiplying by $T^{-t}$ we obtain $\boldsymbol{T}^{-\boldsymbol{t}} \boldsymbol{\sigma}=C^{*}(\boldsymbol{T} \epsilon)$. If we now define the "mapped" quantities $\sigma^{*}=T^{-t} \sigma$ and $\epsilon^{*}=T \epsilon$ we can observe that the relation $\sigma^{*}=C^{*} \epsilon^{*}$ is isotropic, that is, that the matrix $\boldsymbol{T}$ defines the mapping operator we were looking for. $C$ can be approximated by taking into account the results from

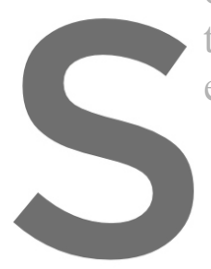
the RVE analysis. Valu each virtual experiment.
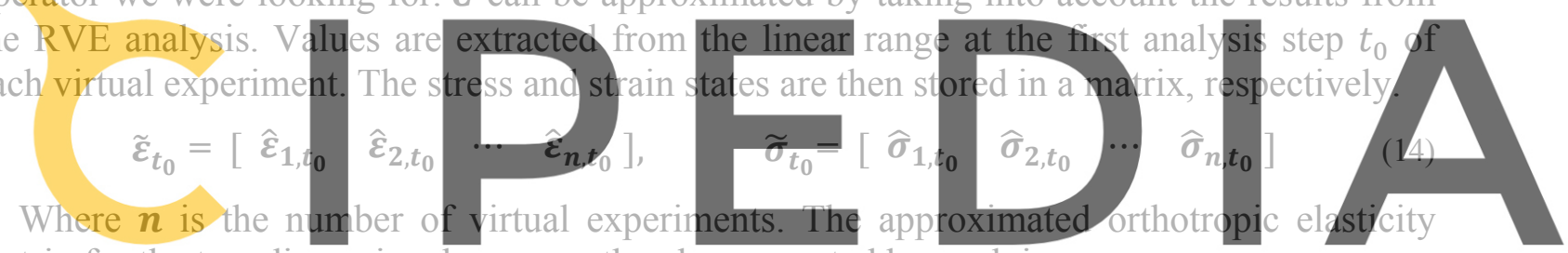

matrix for the two dimensional case can then be computed by applying

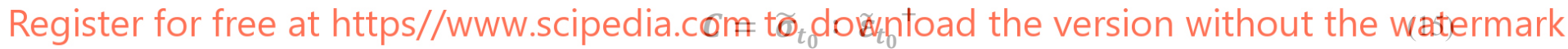

Where $\widetilde{\varepsilon}_{t_{0}}{ }^{+}$is the Moore-Penrose Inverse of $\widetilde{\varepsilon}_{t_{0}}$. The application of Equation (12) requires that the number of virtual experiments $\boldsymbol{n}>1$, by definition the Moore Penrose Inverse is supporting the least squares solution to the problem $\widetilde{\boldsymbol{\sigma}}_{\boldsymbol{t}_{\mathbf{0}}}=\boldsymbol{C} \widetilde{\boldsymbol{\varepsilon}}_{\boldsymbol{t}_{\mathbf{0}}}$.

In order to define $\boldsymbol{C}^{*}$ as the isotorpic matrix, the research presented here considers a optimization procedure that finds the closest isotropic matrix to an orthotropic one by searching the minimum of the Frobenius norm of both the isotropic and the orthotropic matrix. This optimization includes the modification of the isotropic elastic constants $\mathrm{E}$ and $v$.

The machine-learning procedure is then performed according to the following steps: i) computation of the anisotropic elasticity matrix $\boldsymbol{C}$ by Moore Penrose; ii) computation of the closest isotropic matrix $\boldsymbol{C}^{*}$; iii) computation of the transformation matrix $\boldsymbol{T}$; iv) mapping of the training data to the isotropic level; v) train the isotropic constitutive damage model.

Once then isotropic constitutive model is trained, in order to use it, one would follow the steps: i) given the anisotropic strains $\boldsymbol{\epsilon}$ compute the isotropic strains $\boldsymbol{\epsilon}^{*}$; ii) apply the trained constitutive law to obtain the isotropic stress $\boldsymbol{\sigma}^{*}$; iii) map $\boldsymbol{\sigma}^{*}$ onto $\boldsymbol{\sigma}$ into the real anisotropic space. 


\section{APPLICATION}

This Section presents the application of the machine-learning homogenization technique to a two dimensional masonry wall. Figure 3 a shows the RVE of the virtual laboratory and its boundary. The wall is build up by bricks of size $0.309 \mathrm{~m} \times 0.1475 \mathrm{~m} \times 0.045 \mathrm{~m}$. The thickness of the vertical and horizontal mortar joints is $0.012 \mathrm{~m}$. These dimensions allow a spatial allocation of the bricks and mortar equal to the "Flemish Bond". The RVE's total dimensions are $0.53 \mathrm{~m}$ in $\mathrm{x}$ - and $\mathrm{y}$-directions, respectively.

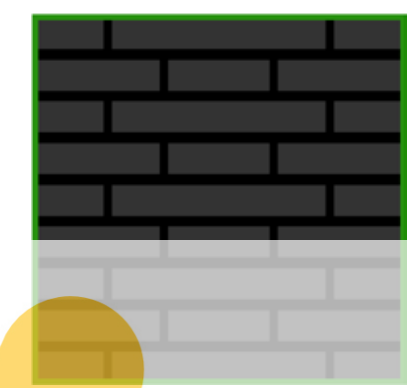

a)

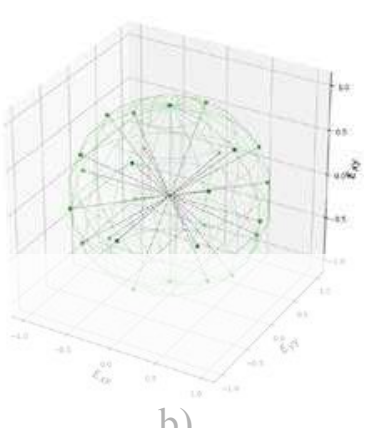

b)

Figure 3: Virtual laboratory: a) Micro model of the representative volume element showing the brick units, the mortar joints and the boundary; b) generated strain database for the boundary displacement application

The FE modelling was carried out by utilizing the program GiD Pre- and Postprocessor [24]. The average elemer elements in its thickness integration have been use

The FE mesh is passed to brick units and mortar ent size counts 0.006
sed for the FE mesh. Th
sed to the python based
joints are assigned with $m$ so that the no
ally quadrilatera
he total number
FE program $K$

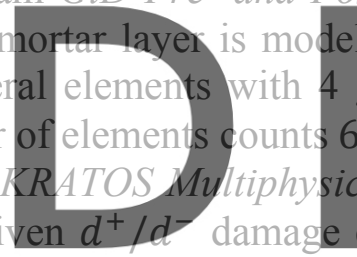
gauss point
6160.
cs. Both the model introduced in Section 3. Table 1 shows the properties assigned to both the materials.

Register for free at https//www.scipedia.com to download the version without the watermark Table 1. Material data for the brick units and the mortar joints applied to the masonry RVE

\begin{tabular}{c||ccccccccccccccc} 
& $\boldsymbol{E}$ & $\boldsymbol{v}$ & $\boldsymbol{f}_{\boldsymbol{p}}^{+}$ & $\boldsymbol{G}^{+}$ & $\boldsymbol{f}_{\mathbf{0}}^{-}$ & $\boldsymbol{f}_{\boldsymbol{p}}^{-}$ & $\boldsymbol{f}_{\boldsymbol{k}}^{-}$ & $\boldsymbol{f}_{\boldsymbol{r}}^{-}$ & $\boldsymbol{G}^{-}$ & $\boldsymbol{\varepsilon}_{\boldsymbol{p}}^{-}$ & $\boldsymbol{c}_{\mathbf{1}}^{-}$ & $\boldsymbol{c}_{\mathbf{2}}^{-}$ & $\boldsymbol{c}_{\mathbf{3}}^{-}$ & $\boldsymbol{k}_{\boldsymbol{b}}$ & $\boldsymbol{\kappa}$ \\
& {$[G P a]$} & {$[-]$} & {$[M P a]$} & {$[N / m]$} & {$[M P a]$} & {$[M P a]$} & {$[M P a]$} & {$[M P a]$} & {$[N / m]$} & {$\left[10^{-2}\right]$} & {$[-]$} & {$[-]$} & {$[-]$} & {$[-]$} & {$[-]$} \\
\hline \hline Brick & 7.0 & 0.2 & 1.5 & 48.0 & 2. & 6.0 & 4.6 & 2.0 & 3600.0 & 1.0 & 0.65 & 0.5 & 1.5 & 1.2 & 0.16 \\
Mortar & 1.8 & 0.2 & 0.15 & 20.0 & 2.0 & 6.0 & 4.6 & 2.0 & 3600.0 & 1.0 & 0.65 & 0.5 & 1.5 & 1.2 & 0.16
\end{tabular}

For the generation of boundary conditions 26 cases are considered. Figure $3 \mathrm{~b}$ displays the database of strain states $\boldsymbol{\varepsilon}$ for the virtual laboratory (generated from Equation (10)). A single virtual experiment then consists of a nonlinear analysis of the RVE by monotonically increasing the displacement configuration.

By applying the mapping procedure of Section 5 the transformation matrices and the isotropic linear elastic material data for the homogenized material can be derived. The linear elastic isotropic constants are $E=4.46 \mathrm{GPa}$ and $=0.187$.

After having finalized the entire procedure explained in Section 5, the training of the machine-learning model starts. In order to simplify the machine-learning procedure the entire 
training set is split into three sets. Then a 3 phased optimization can be performed by only training selected variables that correlate to the material behaviour represented in each set. Each set trains selected variables only in its assigned training set. Figure 4 shows the training progression of each variable during the optimization, furthermore it shows the training loss. Table 2 shows the optimized results for the parameters of a homogenized material model for masonry.
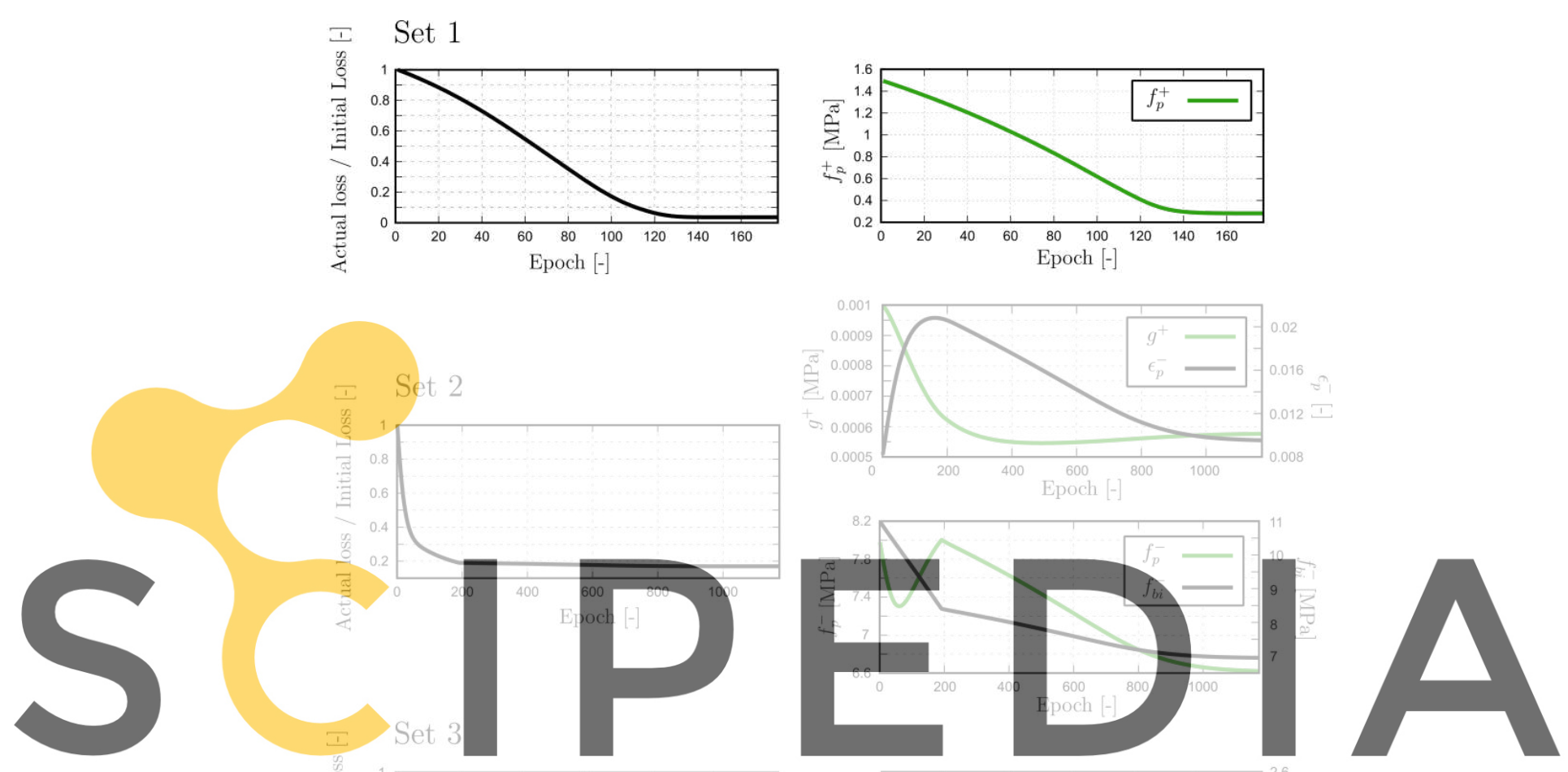

Register for free at https/Xwww.scipedia.com to download the version without the watermark
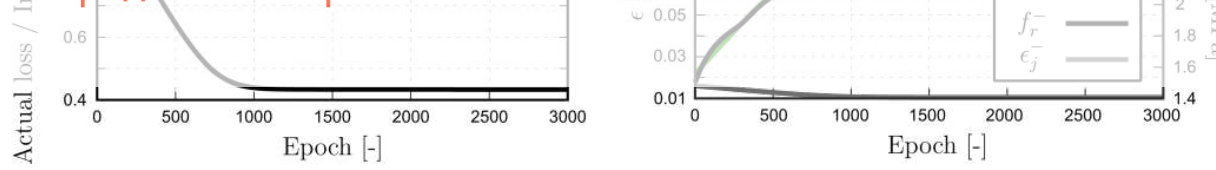

Figure 4: Example of a micro-scale modelled representative volume element $\boldsymbol{\Omega}_{\boldsymbol{R} V \boldsymbol{E}}$ for the virtual laboratory with boundary $\partial \boldsymbol{\Omega}_{R V E}$

Table 2: Values obtained from the machine-learning optimization technique at the isotropic level for a masonry wall with bricks allocated according to the Flemish bond

\begin{tabular}{|c|c|c|c|c|c|c|c|c|c|c|}
\hline \multirow{3}{*}{$\begin{array}{l}\text { Homogenized } \\
\text { Constitutive } \\
\text { Model }\end{array}$} & $f_{p}^{+}$ & $G^{+}$ & $f_{0}^{-}$ & $f_{p}^{-}$ & $f_{b i}^{-}$ & $f_{r}^{-}$ & $\varepsilon_{p}^{-}$ & $c_{1}^{-}$ & $c_{2}^{-}$ & $c_{3}^{-}$ \\
\hline & {$[\mathrm{MPa}]$} & {$[\mathrm{N} / \mathrm{mm}]$} & {$[\mathrm{MPa}]$} & {$[\mathrm{MPa}]$} & {$[\mathrm{MPa}]$} & {$[\mathrm{MPa}]$} & {$[-]$} & {$[-]$} & {$[-]$} & {$[-]$} \\
\hline & 0.28 & 0.30 & 1.0 & 6.62 & 6.95 & 2.45 & 0.0095 & 0.5 & 0.06 & 7.53 \\
\hline
\end{tabular}

\section{CONCLUSIONS}

This research has presented a novel homogenization technique for heterogeneous materials. The method deviates from classical homogenization procedures by utilizing a 
machine-learning model. Machine-learning model can detect the link between coupled inputs and outputs by searching it in a mathematical formulation. In this work, the mathematical formulation is constricted to be a strain driven non-linear constitutive law.

This research has introduced a virtual laboratory for training data production. In the virtual laboratory a representative volume element (RVE) of the considered heterogeneous material is built up. While running a large amount of different nonlinear numerical analyses on the RVE, data are stored as coupled strain and stress states in order to train the parameters of a machine-learning model. The procedure also respects the orthotropic material behaviour by applying a mapping transformation from an orthotropic to an isotropic state. The presented procedure is applied to a two dimensional masonry wall. A properly constructed micro model RVE of the wall was constructed as a finite element model. A large amount of different boundary conditions were applied to the nonlinear analysis of the RVE in the virtual laboratory. The machine-learning model of the application case is a properly defined nonlinear $d^{+} / d^{-}$damage constitutive law that has shown to present the brittle behaviour of masonry structures efficiently. At the optimization stage, the parameters of the constitutive law were trained in order to be able to predict the stress states as approximately as possible.

The future step of this research is the implementation of the trained constitutive law to a FE software. This also includes the transformation procedure. Then the applicability and the accuracy of the trained model can be tested for the analysis at the macro scale.

Acknowledgements. The authors gratefully acknowledge the financial support from the Ministry of Science, Innovation and Universities (MCIU) of the Spanish Government, the State Agency of Research (AEI) and the European Regional Development Fund (ERDF) through the SEVERUS project ("Multilevel evaluation of seismic vulnerability and risk mitigation of masonry buildings in resilient historical urban centres", ref. num. RTI2018099589-BI00). Support from Secretaria d'Universitats i Investigació de la Generalitat de Catalunya through a predoctoral grant awarded to the first author is gratefully acknowledged.

\section{REFERENCES}

[1] Oliveira, D.V. and Lourenço, P.B. Implementation and Validation of a constitutive model for the cyclic behaviour of interface elements, Computers and Structures, vol.28 (2004), pp.1451-1461

[2] Drougkas, A., Roca, P. and Molins, C. Analytical micro-modellingof masonry periodic unit cells - Elastic properties, International Journal of Solids and Structures,vol.69-70 (2015), pp.169-188

[3] Petracca, M., Pelà, L., Rossi, R., Zaghi, S., Camata, G. and Spacone, E. Micro-scale Continuos and Discrete Numerical Models for Nonlinear Analysis of Masonry Shear Walls, Construction and Building Materials, vol. 149 (2017), pp. 296-314

[4] Saloustros, S., Pelà, L., Roca, P. and Portal, J. Numerical analysis of Structural Damage in the Church of the Poblet Monastery, Engineering Failure Analysis, vol. 48 (2015), pp.41-61

[5] Lotfi, H. and Shing, P. An Appraisal of Smeared Crack Models for Masonry Shear Wall Analysis, Computers \& Structures, vol. 41 (1991), pp.413-425

[6] Pelà, L., Cervera, M. and Roca, P An orthotropic damage model for the analysis of 
masonry structures, Construction and Building Materials, vol. 41 (2013), pp.957-967[12]

[7] Kalkbrenner, P., Pelà, L. and Sandoval, C. Multi Directional Pushover Analysis of Irregular Masonry Buildings without Box Behaviour, Engineering Structures, vol. 201 (2019)

[8] Petracca, M., Pelà, L., Rossi, R., Oller, S., Camata, G. and Spacone, E. Regularization of first order computational homogenization for multi-scale analysis of masonry structures, Computational Mechanics, vol.57 (2016), no.2, pp.257-276

[9] Petracca, M., Pelà, L., Rossi, R., Oller, S., Camata, G. and Spacone, E. Multiscale computational first order homogenization of thick shells for the analysis of out-of-plane loaded masonry walls, Computer Methods in Applied Mechanics and Engineering, vol. 315 (2017), pp.273-301

[10] Logarzo H.J., Capuano G., Rimoli J.J., Smart constitutive laws: Inelastic homogenization through machine-learning, Computer methods in applied mechanics and engineering, vol. 373 (2021)

[11] Zaghi, S., Martinez, M., Rossi, R. and Petracca, M. Adaptive and Off-line Techniques for Non-linear Multiscale Analysis, Composite Structures, vol. 206 (2018), pp.215-233

[12] Woody Ju, J.W., Chaboche, J.L. and Voyiadjis, G.Z. Damage Mechanics in Engineering Materials. Elsevier Science Ltd., 1998.

[13] Cervera, M., Oliver, J. and Faria, R. Seismic Evaluation of concrete dams via continuum damage models. International Journal of Solids and Structures, vol.24 (1995), no.9, pp. 1225-1245.

[14] Faria, R., Oliver, J. and Cervera, M. A strain-based plastic viscous-damage model for massive conscrete structures, International Journal of Solids and Structures, vol.43(1998), no.14, pp.1533-1558.

[15] Wu, J.Y., Li, J. and Faria, R. An energy release rate-based plastic damage model for concrete, International Journal of Solids and Structures, vol.43(2006), no.3-4, pp.583-612

[16] Faria, R., Oliver, J. and Cervera, M. An Isotropic Scalar Damage Models for the Numerical Analysis of Concrete Structures, CIMNE Monograph, vol December (2000), no. 198

[17] Lubliner, J., Oliver, J., Oller, S. and Oñate, E. A plastic damage model for concrete, International Journal of Solids and Structures, vol.25 (1989), no.3, pp.299-326

[18] Ruder, S. An overview of gradient descent optimization algorithms, arXiv preprint arXiv:1609.04747 (2016)

[19] Kingma, D.P. and Lei Ba, J. Adam: A Method For Stochastic Optimization, paper submitted to: International Conference on Learning Representations (2015)

[20] https://www.tensorflow.org/

[21] Dadvand, P., Rossi, R. and Oñate, E. An Object-oriented Environment for Developing Finite Element Codes for Multi-disciplinary Applications, Archives of Computational Methods in Engineering, vol.17 (2010), no.2, pp.253-297

[22] Norris A., The isotropic material closest to a given anisotropic material, Journal of Mechanics of Materials and Structures 1 (2005)

[23] Rossi R., Zorilla R., Codina R., A stabilised displacemen-volumetric strain formulation for nearly incompressible and anisotropic materials, CMAME, 2020

[24] Melendo A., Coll A., Pasenau M., Escolano E., Monros A., www.gidhome.com, (2016) 